Rapid Reviews COVID-19

\title{
Reviews of "Firearm
}

Purchasing and Firearm

Violence in the First

Months of the Coronavirus

Pandemic in the United

States"

Jared D. Fisher ${ }^{1}$, David W. Puelz ${ }^{2}$

${ }^{1}$ Department of Statistics, University of California, Berkeley,

${ }^{2}$ Booth School of Business, University of Chicago

Published on: Nov 23, 2020

DOI: $10.1162 / 2 \mathrm{e} 3983 f 5.23029 a 86$

License: Creative Commons Attribution 4.0 International License(CC-BY 4.0). 
To read the original manuscript, click the link above.

Summary of Reviews: This pre-print shows an association between firearm purchases and firearm violence during the pandemic; however, clarification is needed about the soundness of the methods used and how to elucidate further the causality at play.

Reviewer 1 (Jared D. Fisher, David W. Puelz)

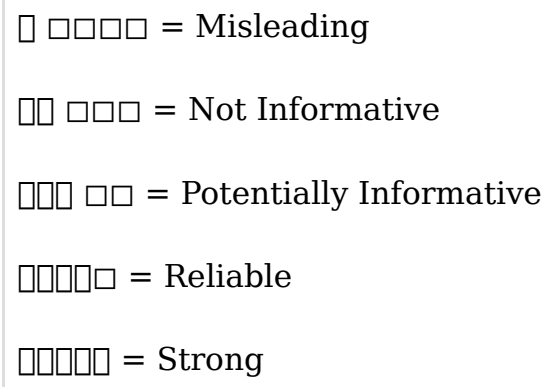

To read the reviews, click the links below. 